\title{
Interfacial engineering manipulation of magnetic anisotropy evolution via orbital reconstruction in low-dimensional manganite superlattices
}

\author{
Guowei Zhou ${ }^{1,2 \dagger}$, Huihui $\mathrm{Ji}^{1 \dagger}$, Zhi Yan ${ }^{1}$, Miming Cai ${ }^{4}$, Penghua Kang ${ }^{1}$, Jun Zhang ${ }^{1}$, Jingdi Lu ${ }^{4}$, \\ Jinxing Zhang ${ }^{4}$, Jingsheng $\mathrm{Chen}^{3^{*}}$ and Xiaohong $\mathrm{Xu}^{1,2^{*}}$
}

\begin{abstract}
Control of magnetic anisotropy in low-dimensional systems is of paramount importance in terms of their fundamental and technological perspectives. $\mathrm{La}_{0.7} \mathrm{Sr}_{0.3} \mathrm{MnO}_{3}$ (LSMO) is a ferromagnetic half-metal with a high Curie temperature and many efforts have been made to control its magnetic anisotropy. However, the relationship between the evolution of the magnetic anisotropy orientation and the electronic structure of low-dimensional LSMO still remains poorly understood. Here, the high-quality superlattices comprised of LSMO and $\mathrm{SrMnO}_{3}$ (SMO) layers are synthesized with a compatible structure at the atomic scale. Their magnetic anisotropy is gradually varied from planar to perpendicular by increasing the SMO thickness, and the special fourfold magnetic anisotropy is also observed at the intermediate superlattice thickness. The evolution of the magnetic anisotropy in these systems is confirmed by the electronic transport and magnetic measurements. Moreover, X-ray linear dichroism measurements and first-principles calculations reveal the interfacial orbital reconstruction with the in-plane to out-ofplane magnetic reorientation transition. Therefore, a new microscopic method for magnetic anisotropy manipulation is developed in the present study, enabling discovery of novel phenomena as well as control of the magnetic properties.
\end{abstract}

Keywords: perpendicular magnetic anisotropy, magnetic measurement, transport measurement, interfacial engineering, orbital reconstruction

\section{INTRODUCTION}

Magnetic anisotropy, determining the preferred direction of the magnetic moment in magnetic materials, is one of the most important fundamental properties for various applications such as magnetic memory and logic devices [1-3]. Particularly, perpendicular magnetic anisotropy (PMA) is essential for the design of appliances with high density, high stability, and low energy consumption [4,5]. In this respect, $\mathrm{La}_{0.7} \mathrm{Sr}_{0.3} \mathrm{MnO}_{3}$ (LSMO) with a high Curie temperature is known for its outstanding properties including colossal magnetoresistance and half metallic characteristics with nearly $100 \%$ spin polarization, which are highly demanded in all-oxide spintronics [6-9]. However, LSMO possesses a preferred in-plane (IP) orientation of the magnetic easy axis [10-13]. Until now, only three extrinsic methods can slightly change the easy axis direction of LSMO films from IP to out-of-plane (OOP) [14-20]. (1) Mechanical strain: the fabrication of compressively strained LSMO films on $\mathrm{LaAlO}_{3}$ substrate have resulted in the PMA behavior and the formation of the unprecedented $\mathrm{LaSrMnO}_{4}$ impurity phase due to large lattice mismatch $[4,5,21]$; (2) crystal structural evolution: the tunable oxygen octahedral rotation induced by the modification of crystal structure results in the PMA state in doping $\mathrm{La}_{1-x} \mathrm{Sr}_{x} \mathrm{MnO}_{3} / \mathrm{SrIrO}_{3}$ superlattices [17]; (3) symmetry mismatch: the symmetry mismatch induced the IP to OOP magnetic easy axis reorientation in $\mathrm{LSMO} / \mathrm{LaCoO}_{2.5}$ (perovskite/ brownmillerite) [16]. However, in these three cases, the interfacial structure failure is inevitable because of the mechanical strain, crystal structural evolution, or symmetry mismatch. These characteristics make the ultrathin LSMO-based heterostructures unfavorable for spintronics applications. In addition, the relationship between the evolution of magnetic easy axis and the electronic structure in low-dimensional LSMO ultrathin films remains elusive. Therefore, the detailed study of magnetic anisotropy in ultrathin LSMO-based heterostructures is significantly important for potential interfacial engineering purposes.

Artificial oxide heterostructures with chemically abrupt interfaces provide a platform for exploring emergent phenomena [22-25]. The engineered interfacial interactions among the lattice, spin, charge, and orbital degrees of freedom are found to play a direct and crucial role in the properties of $3 \mathrm{~d}$ transition oxide materials [26-30]. In the present work, a series of structure compatible $\mathrm{LSMO}(4) / \mathrm{SrMnO}_{3}(n)$ superlattices were deposited onto the (001)-oriented $\mathrm{SrTiO}_{3}$ (STO) substrates. These two kinds of materials have a compatible spin-orbital coupling (SOC), the same symmetry (both are perovskite structure), and similar oxygen octahedral rotation. Compared with $\mathrm{LSMO} / \mathrm{SrIrO}_{3}$ superlattices, $\mathrm{SrMnO}_{3}(\mathrm{SMO})$ is an insulator. Therefore, LSMO is electrically confined in a well and magne-

\footnotetext{
${ }^{1}$ School of Chemistry and Materials Science of Shanxi Normal University \& Key Laboratory of Magnetic Molecules and Magnetic Information Materials of Ministry of Education, Linfen 041004, China

${ }^{2}$ Research Institute of Materials Science of Shanxi Normal University \& Collaborative Innovation Center for Shanxi Advanced Permanent Magnetic Materials and Technology, Linfen 041004, China

${ }^{3}$ Department of Materials Science and Engineering, National University of Singapore, Singapore 117575, Singapore

${ }^{4}$ Department of Physics, Beijing Normal University, Beijing 100875, China

${ }^{\dagger}$ These authors contributed equally to this work.

* Corresponding authors (emails: msecj@nus.edu.sg (Chen J); xuxh@sxnu.edu.cn (Xu X))
} 
tically decoupled between adjacent LSMO layers with increasing SMO thickness. Therefore, the extrinsic influence factors, such as mechanical strain, crystal structural evolution, and symmetry mismatch, can be excluded, and the intrinsic interfacial interactions between the degrees of freedom are found to play a crucial role in the properties of $3 \mathrm{~d}$ transition oxide materials. The magnetic anisotropy is gradually varied from planar to perpendicular by increasing the SMO thickness, and the special four-fold magnetic anisotropy is also observed at the intermediate superlattice thickness. The evolution of the magnetic anisotropy in these systems is confirmed by both the electronic transport and magnetic measurements. X-ray linear dichroism (XLD) and density functional theory (DFT) calculations confirmed the interfacial orbital reconstruction from the IP to OOP magnetic easy-axis changes, which was found to be consistent with the Bruno's model [31]. Therefore, the gradual control of the magnetic easy axis reorientation is achieved in the present study through a simple control of the SMO layer dimensionality.

\section{EXPERIMENTAL SECTION}

\section{Sample preparation}

Fully epitaxial $\left[(\mathrm{LSMO})_{n} /(\mathrm{SMO})_{n}\right]_{t}$ superlattices were deposited on atomically flat STO (001) substrates pretreated with buffered $\mathrm{HF}$ acid by pulsed laser deposition (PLD). Here $n$ was an integer unit cell (u.c.) varying from 1 to 9 , and $t$ (the thickness of the whole sample in number of LSMO/SMO units) was set at 10. The thickness of LSMO layer was fixed at 4 u.c. For convenience, the LSMO/SMO superlattices were labeled as L4Sn. For comparison, the 40-u.c. single LSMO film and the LSMO/STO superlattice with a similar thickness were grown as well. In addition, the L4S3 superlattice was also deposited on the $\mathrm{LaAlO}_{3}$ substrate. During the deposition, all the high-quality samples were controlled using the in-situ reflection high-energy electron diffraction (RHEED). The distance between the target and the substrate was kept at $75 \mathrm{~mm}$. The samples were deposited at a temperature of $725^{\circ} \mathrm{C}$ using a $248-\mathrm{nm} \mathrm{KrF}$ excimer laser with the frequency of $2 \mathrm{~Hz}$, and oxygen pressure was kept at $100 \mathrm{mTorr}$ $\left(1\right.$ Torr $\left.=1.333 \times 10^{2} \mathrm{~Pa}\right)$. In order to avoid the oxygen vacancies, these samples were in-situ annealed for $1 \mathrm{~h}$ under an oxygen pressure of 300 Torr.

\section{Structural, magnetic, and transport measurement}

The crystal structures of LSMO/SMO superlattices were characterized by a conventional X-ray diffraction (XRD, Ultima IV) technique and a high-resolution four-circle X-ray diffractometer. The scanning transmission electron microscopy (STEM) was performed on a Titan 80-300 microscope equipped with highangle annular dark field (HAADF) to characterize the interfacial microstructure. The IP and OOP magnetic hysteresis loops were measured with vibrating sample magnetometer in a physical properties measurement system (PPMS-VSM). The $\pi / 4$ degree hysteresis loop and the temperature dependence of magnetic moment curves were performed in a superconducting quantum interference device (SQUID) in the temperature range of $5-360 \mathrm{~K}$. The magnetic hysteresis loops were obtained after subtracting the diamagnetic background of plastic tube and STO substrate. The electrical transport measurements were performed using a linear four-probe configuration with the Quantum Design PPMS in the range of 5-300 K. The magnetic field was kept to be perpendicular to the current. The magnetic hysteresis loops and ADMR curves were reproduced on multiple samples.

\section{XAS and XLD measurement}

The X-ray absorption spectroscopy (XAS) of Mn L-edge in LSMO/SMO superlattices was measured at the Beamline BL12Ba of the National Synchrotron Radiation Laboratory (NSRL). The background vacuum level was kept as $5 \times 10^{-7}$ Torr and the absorption signal was detected by the total electron yield (TEY) method. The XAS spectra were normalized in order that the $\mathrm{L}_{3}$ and $\mathrm{L}_{2}$ edges have coincident intensities for the two polarized directions. After that, the pre-edge spectral region of $\mathrm{L}_{3}$ was defined as zero and the peak at the $\mathrm{L}_{3}$ edge was defined as one. The XLD is described as the difference in the XAS spectra with vertical $(E / / c)$ and horizontal $(E / / a)$ polarizations.

\section{DFT calculation}

The first-principles calculations were performed using the Vienna ab-initio simulation package (VASP) [32]. The generalized gradient approximation (GGA) of Perdew-Burke-Ernzerhof (PBE) type was used to deal with the exchange-related interactions [33]. The cutoff energy was set to $520 \mathrm{eV}$. A $9 \times 9 \times$ 1 gamma-center $k$-point mesh was adopted in the calculations. The convergence criteria for energy and force were defined as $10^{-5} \mathrm{eV}$ and $0.01 \mathrm{eV} \AA^{-1}$, respectively. To describe the strong electron-electron interactions, the GGA $+U$ method with $U=$ $5.0 \mathrm{eV}$ and $J=1.0 \mathrm{eV}$ was considered for Mn $3 \mathrm{~d}$ orbitals as well [34].

\section{RESULTS AND DISCUSSION}

Crystal structure characterization of all manganite superlattices In order to investigate the impact of interfacial engineering on the transport and magnetic properties, the high-quality $\operatorname{LSMO}(4) / \operatorname{SMO}(n)$ (labeled as L4Sn) superlattices were deposited onto STO (001) substrates, where $n$ is the number of unit cells varying from 1 to 9 . The precise control of thickness was achieved by in-situ monitoring of the intensity oscillations of the RHEED patterns during the growth (Fig. S1a), revealing the layer-by-layer growth of both LSMO and SMO constituents. The high quality of the $\mathrm{L} 4 \mathrm{~S} n$ superlattices with controllable thickness at the atomic level was further confirmed by high-resolution XRD and STEM. Fig. 1a displays the XRD profiles of L4Sn superlattices acquired in $\mathrm{Cu}-\mathrm{Ka}$ radiation in the (002) peak range. The emergence of satellite peaks SL \pm 1 in these superlattices indicates their smooth interfaces. The SL -1 peak position decreases with decreasing SMO layer thickness, which follows the Bragg's law and is known as Laue fringes [35]. The obvious Laue fringes are further found to support the flatness of these superlattices. The reciprocal space mapping (RSM) around the (103) reflections was used to confirm the IP high-quality epitaxial growth of L4Sn superlattices. The RSM is a powerful technique for quantifying the strain state, which can be identified by the intensity distribution around the Bragg peak [36]. As shown in Fig. 1b, the characteristic (103) diffraction peaks of L4S1, L4S3, and L4S9 superlattices align vertically with the STO substrates, indicating that these superlattices are under the similar strain from substrates. Hence, these L4Sn superlattices are coherent in both the IP and OOP directions because no diffusion is observed in the transverse or longitudinal direction. To further investigate the interfacial sharpness of L4Sn super- 

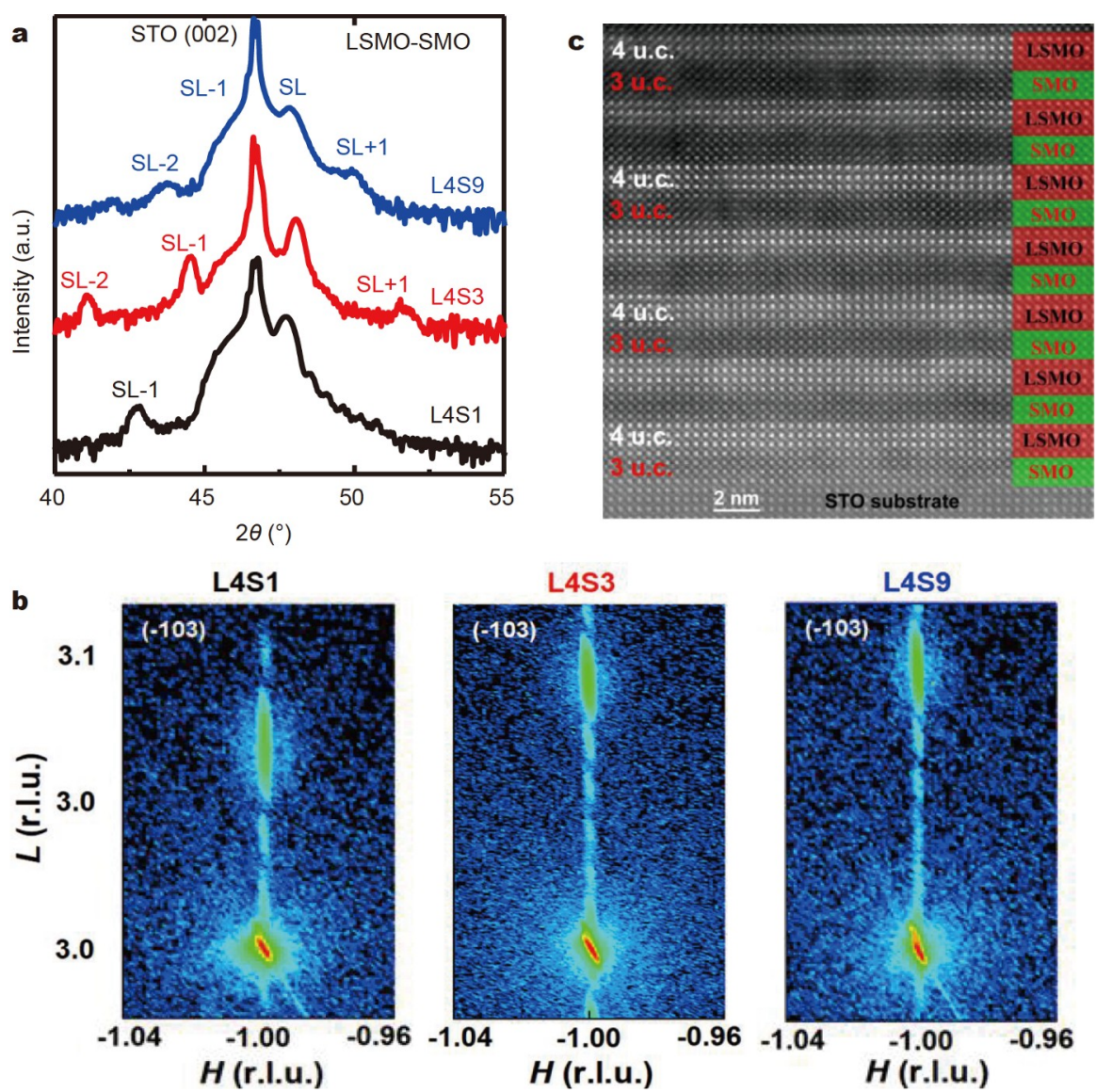

Figure 1 Structural characterization of the LSMO/SMO superlattices. (a) $\theta-2 \theta$ XRD patterns of L4S1, L4S3, and L4S9 superlattices grown on STO substrates. Both the superlattice peaks and the satellite fringes reveal the high degree of interface abruptness. (b) X-ray reciprocal spacing mapping images of the L4S1, L4S3, and L4S9 superlattices around the (103) peaks. The vertical alignment of the reflections suggests the same IP lattice constants of the superlattices as the STO substrates. (c) The partial HAADF STEM image of L4S3 superlattice along the [001] zone axis. The bright and dark regions correspond to LSMO and SMO layers, respectively.

lattices, the atomic-resolved STEM measurements were carried out, as shown in Fig. S1b. The STEM image of L4S3 superlattice is shown in Fig. 1c. Since the image intensity is directly proportional to the atomic number [37], the brighter areas correspond to the LSMO layers, whereas the darker regions represent the SMO layers. It is obvious that the interface is atomically flat. According to the high quality of LSMO/SMO superlattices proved by XRD, RSM, and STEM, the next step of the present study was to elucidate the effect of interfacial engineering on their magnetic and transport properties.

\section{Thickness dependence of the electric transport anisotropy in manganite superlattices}

To monitor the evolution of the magnetic properties in $\mathrm{L} 4 \mathrm{~S} n$ superlattices, angular dependent magnetoresistance (ADMR) measurements were carried out [38,39]. Fig. 2a-c display the polar plots of ADMR of L4S1, L4S3, and L4S9 superlattices measured at $7 \mathrm{~T}$ and the temperatures from 5 to $200 \mathrm{~K}$. The corresponding ADMR curves are also shown in Fig. S2. Here, the ADMR ratio is defined as $\mathrm{ADMR}=\left[R(\theta)-R\left(0^{\circ}\right)\right] / R\left(0^{\circ}\right)$, where $R$ is the resistance and $\theta$ is the angle between the magnetic field and the [100] direction in the film plane [40]. The longitudinal resistance is measured along the [010] direction and the magnetic field is rotated in the (001) plane (Fig. S3). The L4S1 superlattice shows a two-fold IP magnetic anisotropy at all measured temperatures. The sign of MR is similar to that of a single LSMO film grown on the STO substrate (Fig. S6). At the same time, the L4S3 superlattice also demonstrates the two-fold IP easy axis at the temperatures above $10 \mathrm{~K}$, which transforms into the fourfold magnetic anisotropy as the temperature decreases to $5 \mathrm{~K}$. The magnetic easy axes are oriented along the [011], [011], [011], and [011] directions. In turn, the L4S9 superlattice undergoes the parallel-to-perpendicular magnetic anisotropy transition as the temperature decreases from 200 to $5 \mathrm{~K}$. In addition, the fourfold magnetic anisotropy is also observed at the temperatures of 50 and $100 \mathrm{~K}$ (Fig. S2c). Fig. 2d shows the ADMR curves measured at $5 \mathrm{~K}$ for three different $\mathrm{L} 4 \mathrm{~S} n$ superlattices. In particular, the ADMR spectrum at $5 \mathrm{~K}$ under the magnetic field rotating in the (010) and (100) planes is similar to that of the (001) plane (Fig. S3). These results further confirm the existence of PMA in thicker L4Sn superlattices, and the novel fourfold magnetic anisotropic axis (intermediate state) is detected in L4S3 superlattice. In addition, the electric transport anisotropy is also observed in these superlattices with increasing SMO thickness (Fig. S4). The L4S1 superlattice exhibits higher electrical conductivities along the IP direction no matter the $R-T$ is measured under zero field or $5 \mathrm{~T}$ magnetic field. In contrast, for the L4S9 superlattice, the lower resistance 

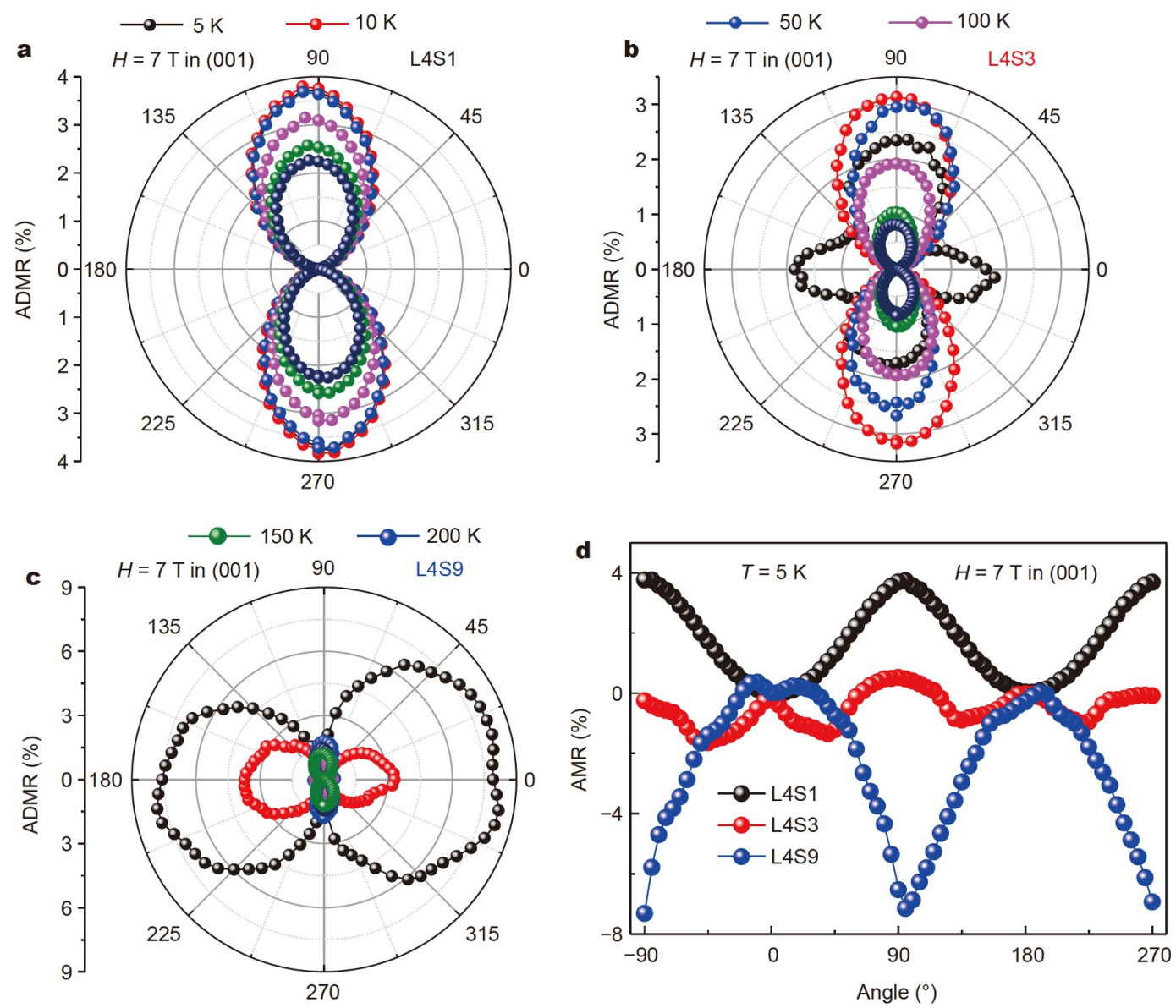

Figure 2 Transport anisotropy of the LSMO/SMO superlattices. The effect of the temperature on the ADMR of (a) L4S1, (b) L4S3, and (c) L4S9 superlattices. All curves are normalized to the resistance at zero degree. The measurement configuration is illustrated in Fig. S3a and the current is along the [010] direction. (d) The ADMR spectra acquired at $5 \mathrm{~K}$ under a magnetic field of $7 \mathrm{~T}$ for the same superlattices.

states are found along the OOP direction under 0 and $5 \mathrm{~T}$ magnetic fields. For L4S3 superlattice, the highly conductive states are undoubtedly along the $\pi / 4$ direction. No thermal hysteresis is found in the cooling down and warming up processes in this experiment [15]. In addition, it is obvious the resistance increases by over 6 orders of magnitude when the $n$ increases from 1 to 9 , showing the thickness-associated metalinsulator transition (Fig. S5). It is known that the SMO material with $\mathrm{Mn}^{4+}$ ions is a band insulator with G-type antiferromagnetic order, and the ferromagnetic and metallic behaviors of LSMO materials are attributed to the double-exchange mechanism between $\mathrm{Mn}^{3+}$ and $\mathrm{Mn}^{4+}$ ions. However, a so-called "dead-layer" thickness, defined as the thinnest layer for metallic and ferromagnetic behaviors, is about $4 \mathrm{~nm}$ (10 u.c.) as LSMO film is grown on the STO substrate [16]. Hence, the transport property should manifest an insulator behavior in these L4S $n$ superlattices. However, in the thinnest L4S1 superlattice, the $\mathrm{Mn}^{4+}$ ions in the SMO layer can connect the neighbor LSMO layer to form the long-range double exchange interaction. This is the reason of the metallic behavior only present in the L4S1 superlattice.

\section{Thickness dependence of the magnetic anisotropy in the} manganite superlattices

Fig. 3a-c display the magnetic hysteresis loops $(M-H)$ along the $\mathrm{IP}$ and OOP directions at $5 \mathrm{~K}$ for L4S1, L4S3, and L4S9 super- lattices, respectively. In the L4S1 superlattice, the magnetic easy axis is obviously in the film plane, as follows from the IP and OOP magnetic curves, which is similar to a single LSMO film (Fig. S6) [10]. While the magnetic easy axis of the L4S9 superlattice is along the OOP orientation with a strong contrast to the observed IP easy axis in a thinner SMO layer, which is consistent with the ADMR results. The microscopic magnetic domain switching, corresponding to the hysteresis of L4S9 superlattice, has also been characterized by using low-temperature magnetic force microscopy (MFM) at $5 \mathrm{~K}$ (Fig. S7). It is obvious that the magnetization is fully aligned in the negative field direction under the saturation field of $-4 \mathrm{~T}$, showing a homogeneous contrast. When the external field is turned towards positive direction, the magnetization starts to reverse with a positive contrast and the homogenous contrast appears at the positive saturated field of $4 \mathrm{~T}[41,42]$. Hence, the magnetic anisotropy switch from the IP to the OOP direction is realized by increasing the SMO layer thickness in the LSMO/SMO superlattices. Owing to the structural compatibility in these L4Sn superlattices, the symmetry mismatch can be effectively avoided in the experiment. Therefore, the magnetic anisotropy tuning is achievable through the intrinsic interfacial engineering of LSMO/SMO superlattices. In addition, it is worth noting that the $M-H$ loop along the IP direction is similar to that in the OOP direction for the L4S3 superlattice, implying the easy axis is along the tilted direction. Fig. $3 \mathrm{~b}$ shows the $45^{\circ} \mathrm{M}-\mathrm{H}$ loop for the L4S3 super- 

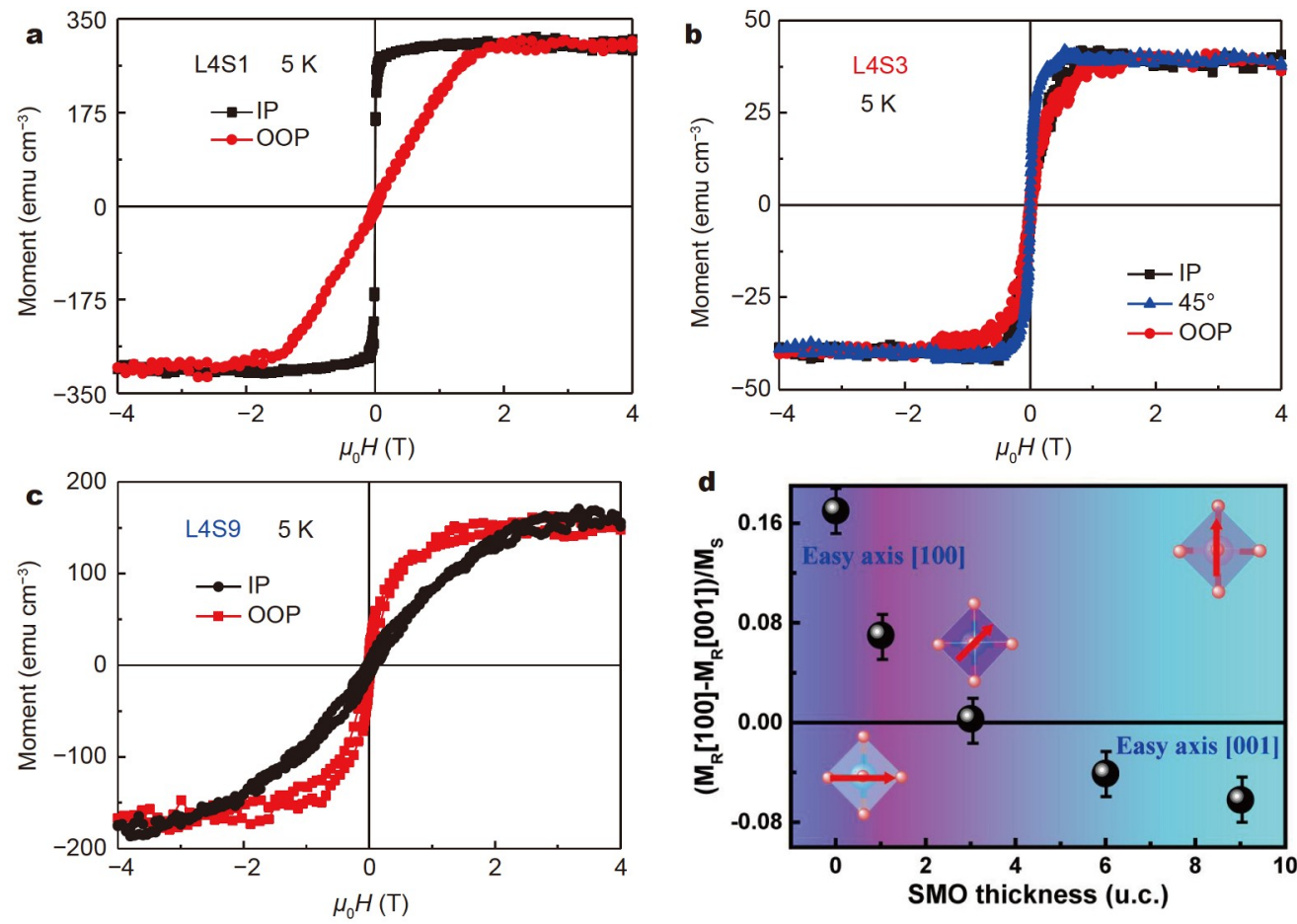

e

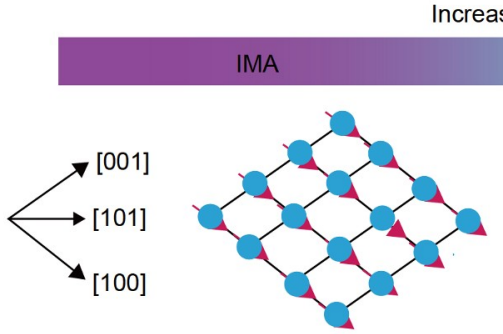

[100] easy axis

ncreasing SMO thickness

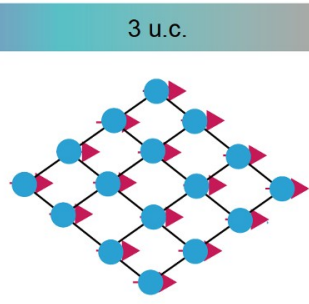

[101] easy axis

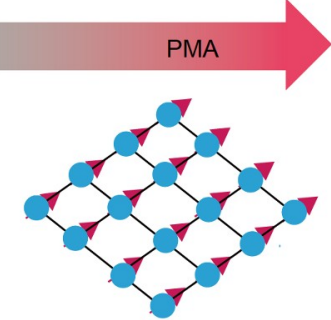

[001] easy axis

Figure 3 Magnetic anisotropy of the LSMO/SMO superlattices. Magnetic hysteresis loops at $5 \mathrm{~K}$ along the IP (black) and OOP (red) orientations for (a) L4S1, (b) L4S3, and (c) L4S9 superlattices. The $45^{\circ}$ ([101]) direction magnetic hysteresis loop is observed for the L4S3 superlattice in (b). (d) Magnetic anisotropy as a function of SMO thickness $(n)$ in a series of L4S $n$ superlattice. Here, MA is defined as the difference of remnant moment $\left(M_{\mathrm{R}}\right)$ between two crystallographic directions normalized by the saturation moment $\left(M_{\mathrm{S}}\right)$. The positive signal corresponds to the IP easy axis, whereas the negative signal indicates the OOP easy axis. The dot at $n$ as zero shows the anisotropy of a single LSMO film. The magnetic easy axis is shown by the arrow in the oxygen octahedral for the series of L4Sn superlattices. Error bars are derived from measurements on multiple samples. (e) The schematics of the corresponding ground states of the Mn spin orientation with increasing SMO layer thickness.

lattice. Compared with IP- and OOP-direction $M-H$ loops, the hysteresis loop of the L4S3 superlattice along the [101] direction is more easily saturated than in the other directions. Therefore, the intermediate state of the easy axis is present in $\mathrm{L} 4 \mathrm{~S} n$ superlattices with increasing thickness of SMO layer. This emergent phenomenon is consistent with the above ADMR results at low temperatures.

The influence of the SMO layer thickness on the magnetic anisotropy is summarized in Fig. 3d, where the normalized difference between the remnant magnetization along the IP and OOP directions is used to represent the change of the magnetic easy axis. The positive and negative signs indicate the IP and OOP easy axes, respectively. It is found that the superlattices with thinner SMO layers $(n<3)$ possess the [100] easy axis consistent with that of the pure LSMO thin film. As the SMO thickness increases $(n>3)$, the easy axis reorients to the [001] direction. When the SMO thickness equals to 3 u.c., the easy axis is at $45^{\circ}$ away from the IP direction. Fig. 3e shows the schematic diagrams of the Mn spin orientations with different thicknesses of SMO layer for better visualization. The easy axis transferring from the IP (L4S1) direction to $45^{\circ}$ (L4S3) with respect to the OOP (L4S9) direction coincides with the thickness variation of transport anisotropy in the ADMR results. To get a quantitative description of the PMA strength, the effective anisotropy constant $\left(K_{\text {eff }}\right)$ was quantitatively estimated by the shadow area between the OOP and IP magnetization curves as shown Fig. S8 [43]. The negative and positive values of $K_{\text {eff }}$ refer to the IP and OOP magnetic anisotropy, respectively. The effective OOP magnetic anisotropy constant of the L4S9 superlattice is as high as $2.14 \times 10^{6} \mathrm{erg} \mathrm{cm}^{-3}\left(1 \mathrm{erg}=10^{-7} \mathrm{~J}\right)$.

The evolution of the magnetic easy-axis direction is further characterized by the temperature dependence of the magnetic moment $(M-T)$ [44]. Fig. 4a, b show the $M-T$ curves under the applied magnetic field along the IP and OOP directions for the L4S1 and L4S9 superlattices, respectively. A typical $M-T$ dependence is observed for the L4S1 superlattice when the 

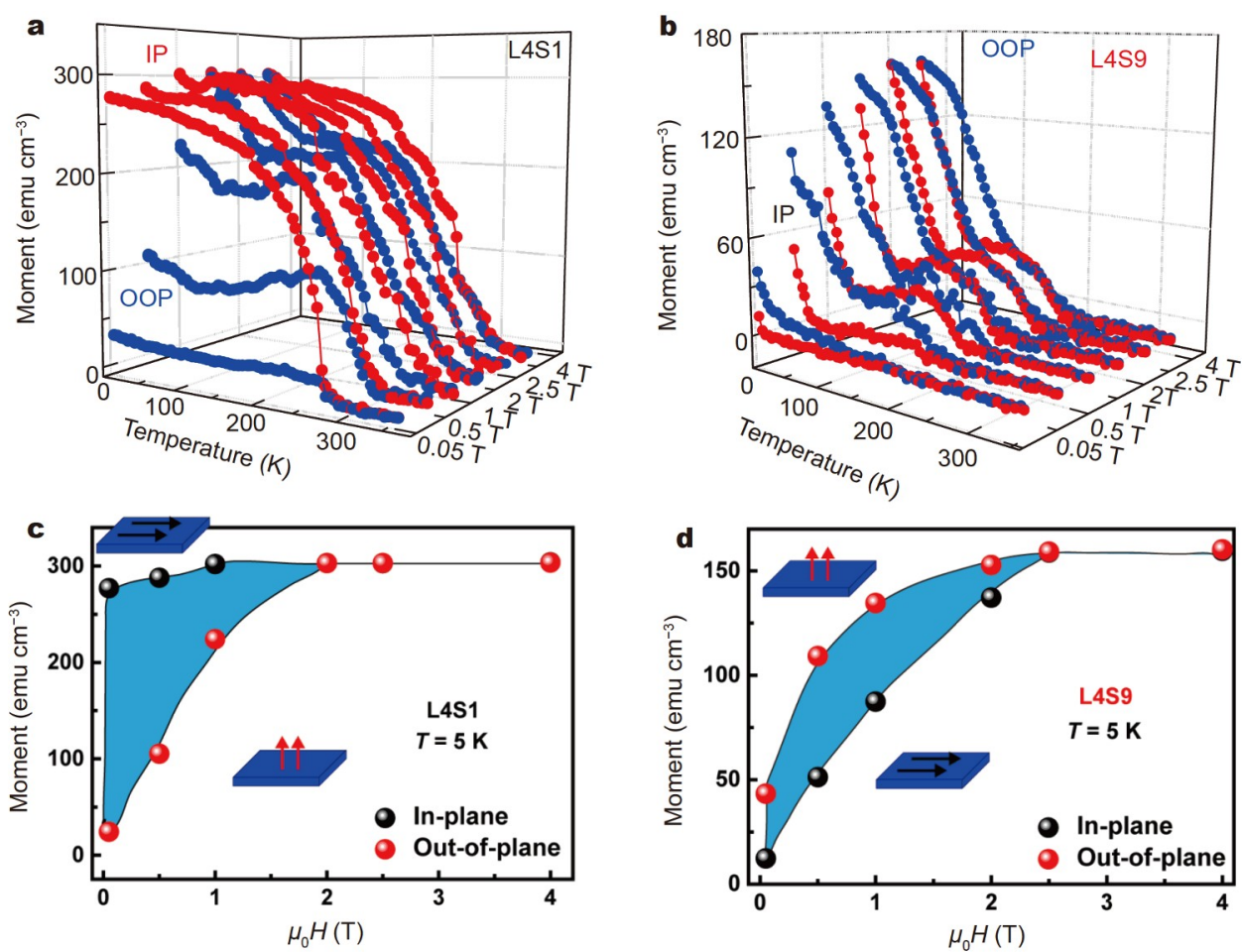

Figure 4 Temperature dependence of the magnetization of LSMO/SMO superlattices. (a, b) Temperature dependence of the magnetization for L4S1 and L4S9 superlattices, respectively. The data were measured in the field-cooling process under IP (red) and OOP (blue) applied fields. (c, d) Magnetization as a function of the applied field obtained from the $M-T$ curves at $5 \mathrm{~K}$. The shaded area corresponds to the energy required to make sure the film plane using magnetic moment.

magnetic field is applied along the sample plane. The magnetic moments reach their maxima at low temperatures, decrease smoothly in the course of heating and rapidly tend to zero at the Curie temperature. These characteristics are similar to those of the LSMO single film (Fig. S6b). In contrast, the magnetic field along the perpendicular direction can only induce substantially low magnetic moments unless it is high enough. Therefore, the easy axis of the L4S1 superlattice is oriented along the IP direction. In sharp contrast to the IP magnetic state of the L4S1 superlattice, an anomalous PMA state is evident from the $M-T$ curves of the L4S9 superlattice. The data measured under the magnetic field of $0.5 \mathrm{~T}$ were afterwards taken as an example. Surprisingly, the IP magnetic moments are low at the low temperature and glossily decrease upon the warming process. On the contrary, the OOP magnetic moments at $5 \mathrm{~K}$ exceed the values along the IP direction by a factor of 2.2. An increase in the temperature causes a slow decrease of the magnetic moments and this superiority remains pronounced up to $180 \mathrm{~K}$.

It is obvious that L4S1 and L4S9 superlattices show the IP and PMA. The magnetizations of these two samples measured at different applied fields are summarized in Fig. 4c, d, respectively. Along the IP orientation, the magnetization of the L4S1 superlattice increases rapidly with the magnetic field and reaches saturation above $0.05 \mathrm{~T}$. In contrast, the magnetization increases gradually, when the field is applied along the OOP direction, which is the typical magnetic moment rotation from the easy axis to a hard axis. In the L4S9 superlattice, the magnetization continuously increase with increasing magnetic field no matter along the IP or the OOP directions until the applied field reaches 2.5 T. Apparently, the OOP direction matches the easy axis for the L4S9 superlattice. Combining these results with the $M-H$ and $M-T$ plots, it is clear that the easy axis varies from the IP to the OOP directions with increasing SMO thickness, and the intermediate state-related $\left(45^{\circ}\right)$ easy axis in L4S3 superlattice is also evident.

\section{Thickness dependence of the orbital reconstruction in the manganite superlattices}

The IP to OOP magnetic anisotropy transition with increasing SMO thickness is comprehensively proved through the above magnetic and transport measurements. Let us now turn to the deeper mechanism for the above to reveal the unique nature of PMA manipulation. It is known that interfacial oxygen octahedral coupling may give rise to orbital reconstruction and thus affect the magnetic anisotropy $[45,46]$. In order to gain more insight into the interfacial coupling effect, the preferential orbital occupation states in these superlattices were characterized by the element selective XAS and XLD measurements [47]. As shown in Fig. 5a, the different linearly polarized photons excite the core electrons, making them occupy the d orbitals. The XLD signal is represented by the intensity difference $\left(I_{a b}-I_{c}\right)$ between the XAS spectra measured with photon polarizations IP $(E / / a)$ and OOP $(E / / c)$. To minimize the impact of the spin momenta on the orbital occupancy, the XLD spectra were recorded at room temperature for all the $\mathrm{L} 4 \mathrm{~S} n$ superlattices. The integrated area of the L-edge of $\mathrm{Mn}$ ions $\left(A_{\mathrm{XLD}}\right)$ was used to represent the difference between the relative occupancies of $x^{2}-y^{2}$ and $3 z^{2}-r^{2}$ orbitals. It is worth noting that, in these LSMO/SMO superlattices, the orbital contribution to the XLD signal is made by mainly the LSMO layer. This is due to the Mn sites in the SMO layer, which 

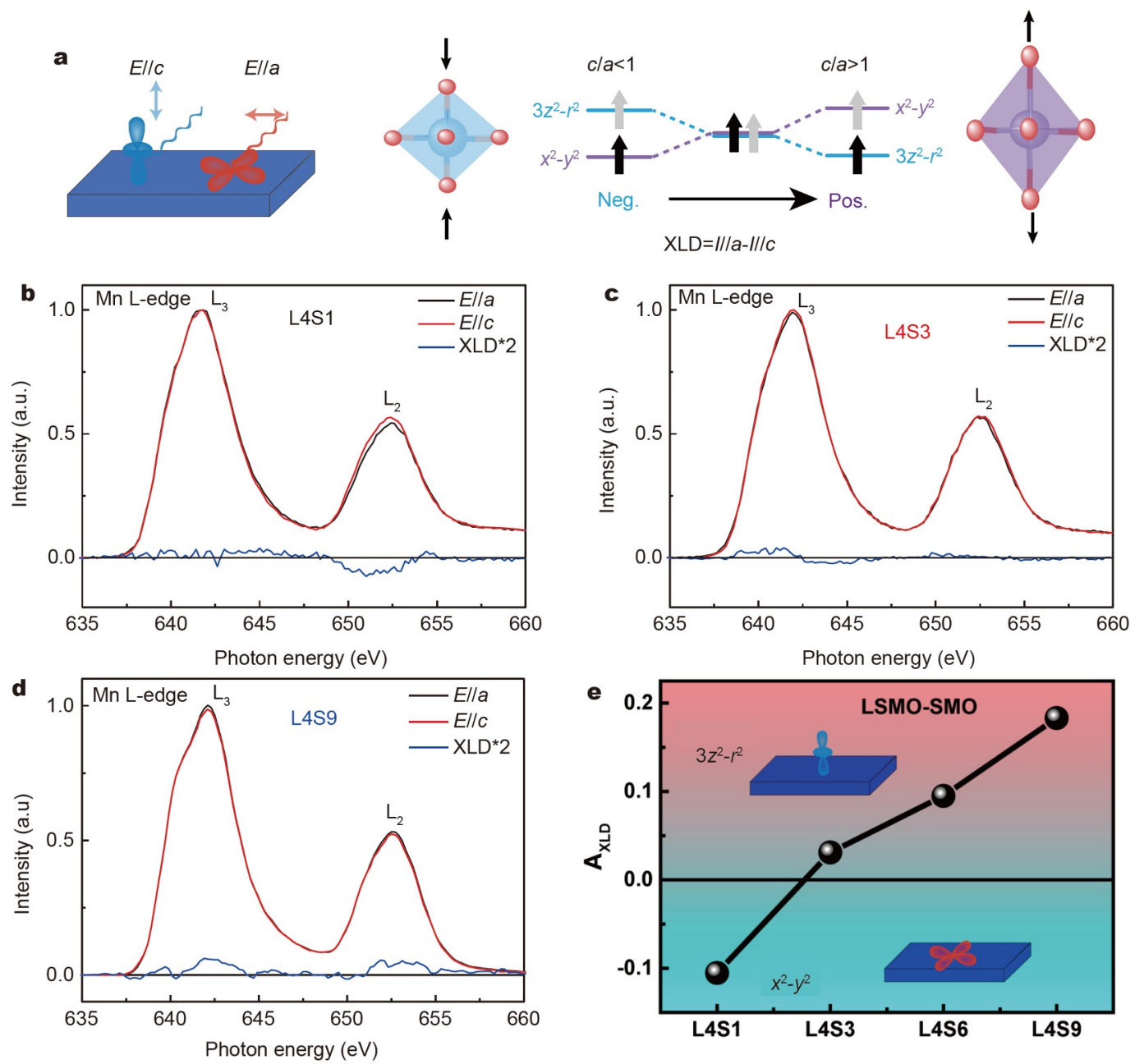

Figure $5 \mathrm{XLD}$ spectra of the LSMO/SMO superlattices. (a) Schematic view of the correlation between XLD and oxygen octahedral deformation. $(\mathrm{b}-\mathrm{d})$ Normalized room temperature XAS spectra of L4S $n$ superlattices at the Mn L-edge. The XLD curves are obtained from the difference between the XAS curves recorded with vertical and horizontal polarizations. For better visualization, the XLD spectra are doubly amplified. (f) Integral area ( $\left.A_{\mathrm{XLD}}\right)$ of the Mn $\mathrm{L}_{2,3}$-edges dependence of L4S $n$ superlattices with the thickness of SMO layer varying from 1 to 9 u.c.

are essentially $3 \mathrm{~d}^{3}$ and cannot significantly influence the XLD signal [48].

Fig. $5 b-d$ show the XLD spectra around the $\mathrm{Mn} \mathrm{L}_{2,3}$-edges along with the IP and OOP XAS spectra for L4S1, L4S3, and L4S9 superlattices, respectively. For the L4S1 superlattice, the $A_{\mathrm{XLD}}$ parameter takes a negative value, indicating that $x^{2}-y^{2}$ orbitals are favorable for occupation rather than the $3 z^{2}-r^{2}$ orbitals. In contrast, for the L4S9 superlattice, the $A_{\text {XLD }}$ value is positive, which suggests a preferential occupancy of the $3 z^{2}-r^{2}$ orbitals. Therefore, the orbital reconstruction takes place in the L4S $n$ superlattices with increasing SMO thickness. As shown in Fig. 5e, the summary of the $A_{\text {XLD }}$ values for different $\mathrm{L} 4 \mathrm{~S} n$ superlattices is presented, revealing the orbital occupancy transition from $x^{2}-y^{2}$ to $3 z^{2}-r^{2}$ orbitals with $n$ increasing from 1 to 9 . In addition, the less orbital occupational difference is found in the L4S3 superlattice although the $A_{\mathrm{XLD}}$ data are two times magnified for better visualization. According to the Bruno's model, the easy axis prefers to be impacted by the direction of orbital momentum [31]. Therefore, the change from IP to OOP magnetic anisotropy in L4Sn superlattices with increasing thickness of SMO layer can be well understood by the orbital reconstruction.

\section{DFT calculations}

In order to further understand these experimental results, the DFT calculation was performed. Fig. 6a displays the computational LSMO/SMO superlattices designed based on the above experimental observations. It is worth mentioning that, in the model construction, the IP lattice constant in the LSMO/SMO superlattices varies with the SMO layer thickness. In particular, the OOP lattice constant $(c)$ changes with the IP lattice parameter while maintaining its original volume. Fig. $6 \mathrm{~b}-\mathrm{e}$ show the spin-resolved projected density of states (PDOS) of $x^{2}-y^{2}$ and $3 z^{2}-r^{2}$ orbitals of the Mn atoms in LSMO/SMO superlattices. As shown in PDOS, both the $x^{2}-y^{2}$ and $3 z^{2}-r^{2}$ orbitals are occupied regardless of the value of $n$. It illustrates that strong lattice distortion of the $\mathrm{MnO}_{6}$ octahedra results in a mixing of the two $\mathrm{e}_{\mathrm{g}}$ orbitals. Through integrating the DOS below the Fermi level, we find the electron occupancy ratio of the Mn $3 z^{2}-r^{2}$ and $x^{2}-y^{2}$ orbitals below the Fermi level varies from $>1$ to $<1$ as the thickness (n) of SMO changes from $n=1,3$ to $n=6,9$. These results mean that, at the smaller SMO thickness, electrons tend 


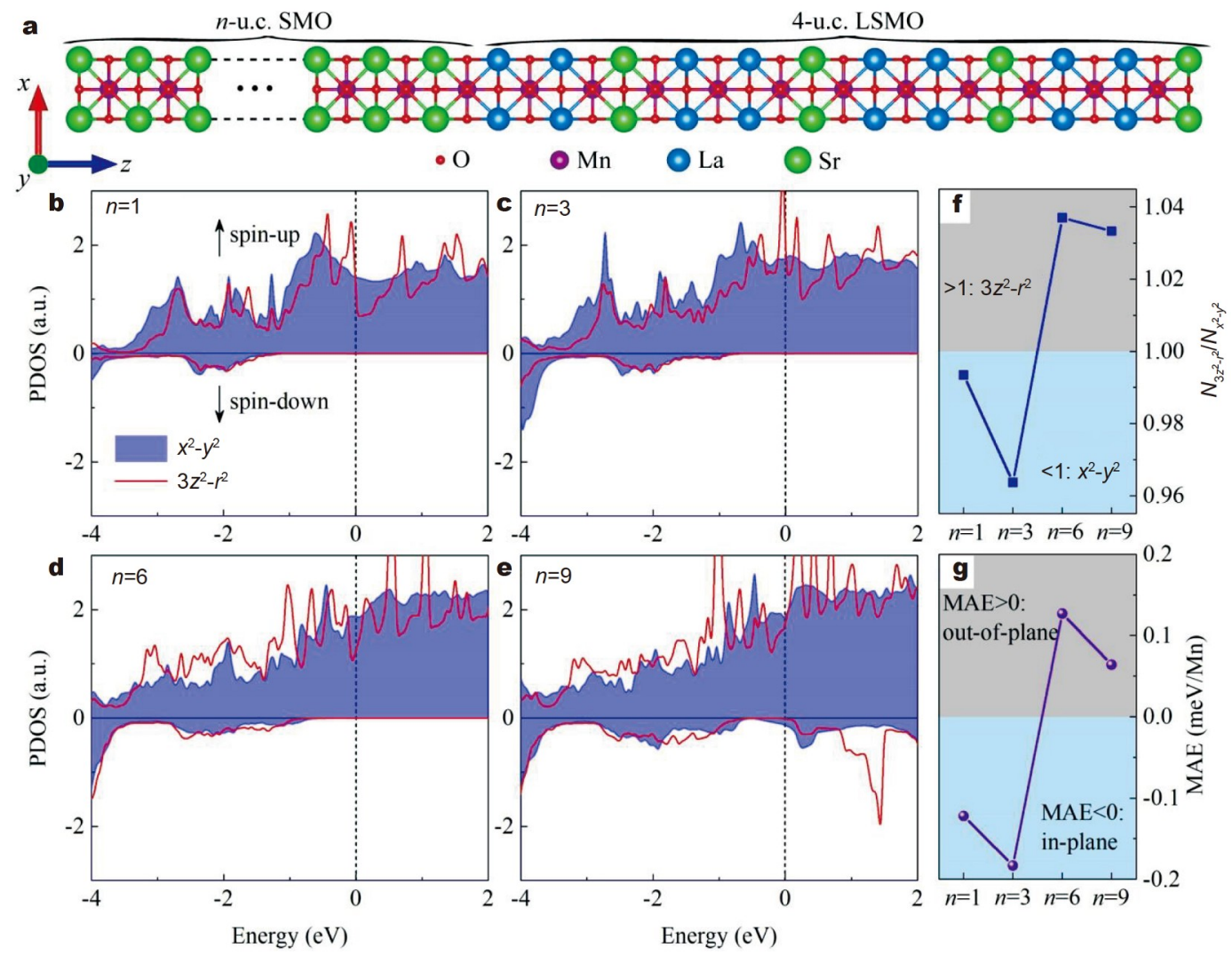

Figure 6 DFT calculations of LSMO/SMO superlattices. (a) Schematic of the LSMO/SMO superlattices with 4-u.c. LSMO and $n$-u.c. SMO ( $n=1,3,6,9)$ layers. (b-e) PDOS of $x^{2}-y^{2}$ and $3 z^{2}-r^{2}$ orbitals of the Mn atoms in LSMO/SMO superlattices. The vertical black dotted lines represent the Fermi level. (f) The evolution of electron occupancy ratio of the Mn $3 z^{2}-r^{2}$ and $x^{2}-y^{2}$ orbitals below the Fermi level for various SMO thicknesses. Here $N_{3 z^{2}-r^{2}}$ and $N_{x^{2}-y^{2}}$ are the electron numbers of $3 z^{2}-r^{2}$ and $x^{2}-y^{2}$ orbitals, respectively. (g) The MAE of LSMO/SMO superlattices.

to occupy $x^{2}-y^{2}$ orbitals, whereas the $3 z^{2}-r^{2}$ orbitals are preferentially filled when using the thicker SMO layers. The aforementioned method of judging the priority order of orbital occupancy by using the electron occupancy ratio was also adopted in previous work [11,49]. Then we turn to the magnetocrystalline anisotropy energy (MAE). With the SOC mechanism, MAE is defined as MAE $=E_{/ /}-E_{\perp}$, where $E_{/ /}$and $E_{\perp}$ denote the total energies corresponding to the integral magnetic moments in the IP and OOP directions, respectively. According to Fig. 6g, the magnetic easy-axis of the LSMO/SMO superlattices gradually shifts from the IP to OOP direction as the thickness of SMO layer increases. Thus, the theoretical results are found to be consistent with the experimental data.

\section{CONCLUSIONS}

In summary, the IP to OOP magnetic anisotropy transition was successfully implemented in the LSMO/SMO superlattices. In particular, the magnetic anisotropy control between the parallel and perpendicular directions was achieved through an increase in the SMO layer thickness. Moreover, the new fourfold magnetic anisotropy state was realized in the L4S3 superlattice. Combining the XLD measurement and DFT calculation results, the interfacial orbital reconstruction at the interface between the LSMO and SMO layers was considered as the main factor of the magnetic anisotropy variation. The transition of the easy axis of magnetic anisotropy from IP in L4S1, the $45^{\circ}$ in L4S3 to OOP in L4S9 is attributed to the orbital reconstruction of $\mathrm{Mn} \mathrm{e}_{\mathrm{g}}$ orbitals.
In L4S1, the electrons preferably occupy the $\mathrm{d}_{x^{2}-y^{2}}$ orbitals, which leads to the IP anisotropy. In L4S9, the electrons preferably occupy the $\mathrm{d}_{3 z^{2}-r^{2}}$ orbitals, which leads to the OOP anisotropy. While in L4S3, the electrons do not show preferable occupations of $\mathrm{d}_{x^{2}-y^{2}}$ orbitals or the $\mathrm{d}_{3 z^{2}-r^{2}}$ orbitals. Therefore, the L4S3 sample shows an effective easy axis of magnetic anisotropy along $45^{\circ}$. Therefore, the findings of this study demonstrate the potential of interfacial coupling in artificial heterostructures, enabling one to discover new phenomena as well as to effectively control the functionalities of such systems.

Received 9 December 2021; accepted 13 January 2022; published online 25 February 2022

1 You L, Lu C, Yang $\mathrm{P}$, et al. Uniaxial magnetic anisotropy in $\mathrm{La}_{0.7} \mathrm{Sr}_{0.3-}$ $\mathrm{MnO}_{3}$ thin films induced by multiferroic $\mathrm{BiFeO}_{3}$ with striped ferroelectric domains. Adv Mater, 2010, 22: 4964-4968

2 Mathews M, Postma FM, Lodder JC, et al. Step-induced uniaxial magnetic anisotropy of $\mathrm{La}_{0: 67} \mathrm{Sr}_{0: 33} \mathrm{MnO}_{3}$ thin films. Appl Phys Lett, 2005, 87: 242507

3 Suzuki Y, Hwang HY, Cheong SW, et al. The role of strain in magnetic anisotropy of manganite thin films. Appl Phys Lett, 1997, 71: 140-142

4 Xiao Z, Zhang F, Farrukh MA, et al. Perpendicular magnetic anisotropy in compressive strained $\mathrm{La}_{0.67} \mathrm{Sr}_{0.33} \mathrm{MnO}_{3}$ films. J Mater Sci, 2019, 54: 9017-9024

5 Cui B, Song C, Wang GY, et al. Strain engineering induced interfacial self-assembly and intrinsic exchange bias in a manganite perovskite film. Sci Rep, 2013, 3: 2542 
6 Fang Z, Solovyev IV, Terakura K. Phase diagram of tetragonal manganites. Phys Rev Lett, 2000, 84: 3169-3172

7 Gibert M, Zubko P, Scherwitzl R, et al. Exchange bias in $\mathrm{LaNiO}_{3-}$ $\mathrm{LaMnO}_{3}$ superlattices. Nat Mater, 2012, 11: 195-198

8 Peng J, Song C, Li F, et al. Charge transfer and orbital reconstruction in strain-engineered ( $\mathrm{La}, \mathrm{Sr}) \mathrm{MnO}_{3} / \mathrm{LaNiO}_{3}$ heterostructures. ACS Appl Mater Interfaces, 2015, 7: 17700-17706

9 Zhou G, Song C, Bai Y, et al. Robust interfacial exchange bias and metal-insulator transition influenced by the $\mathrm{LaNiO}_{3}$ layer thickness in $\mathrm{La}_{0.7} \mathrm{Sr}_{0.3} \mathrm{MnO}_{3} / \mathrm{LaNiO}_{3}$ superlattices. ACS Appl Mater Interfaces, 2017, 9: $3156-3160$

10 Wang B, You L, Ren P, et al. Oxygen-driven anisotropic transport in ultra-thin manganite films. Nat Commun, 2013, 4: 2778

11 Zhang J, Chen X, Zhang Q, et al. Magnetic anisotropy controlled by distinct interfacial lattice distortions at the $\mathrm{La}_{1-x} \mathrm{Sr}_{x} \mathrm{CoO}_{3} /$ $\mathrm{La}_{2 / 3} \mathrm{Sr}_{1 / 3} \mathrm{MnO}_{3}$ interfaces. ACS Appl Mater Interfaces, 2018, 10: 4095140957

12 You L, Wang B, Zou X, et al. Origin of the uniaxial magnetic anisotropy in $\mathrm{La}_{0.7} \mathrm{Sr}_{0.3} \mathrm{MnO}_{3}$ on stripe-domain $\mathrm{BiFeO}_{3}$. Phys Rev $\mathrm{B}, 2013,88$ : 184426

13 Hu S, Cazorla C, Xiang F, et al. Strain control of giant magnetic anisotropy in metallic perovskite $\mathrm{SrCoO}_{3-\delta}$ thin films. ACS Appl Mater Interfaces, 2018, 10: 22348-22355

14 Yi D, Liu J, Hsu SL, et al. Atomic-scale control of magnetic anisotropy via novel spin-orbit coupling effect in $\mathrm{La}_{2 / 3} \mathrm{Sr}_{1 / 3} \mathrm{MnO}_{3} / \mathrm{SrIrO}_{3}$ superlattices. Proc Natl Acad Sci USA, 2016, 113: 6397-6402

15 Liao Z, Huijben M, Zhong Z, et al. Controlled lateral anisotropy in correlated manganite heterostructures by interface-engineered oxygen octahedral coupling. Nat Mater, 2016, 15: 425-431

16 Zhang J, Zhong Z, Guan X, et al. Symmetry mismatch-driven perpendicular magnetic anisotropy for perovskite/brownmillerite heterostructures. Nat Commun, 2018, 9: 1923

17 Yi D, Flint CL, Balakrishnan PP, et al. Tuning perpendicular magnetic anisotropy by oxygen octahedral rotations in $\left(\mathrm{La}_{1-x} \mathrm{Sr}_{x} \mathrm{MnO}_{3}\right) /\left(\mathrm{SrIrO}_{3}\right)$ superlattices. Phys Rev Lett, 2017, 119: 077201

18 Kan D, Aso R, Sato R, et al. Tuning magnetic anisotropy by interfacially engineering the oxygen coordination environment in a transition metal oxide. Nat Mater, 2016, 15: 432-437

19 Zhang B, Wu L, Zheng J, et al. Control of magnetic anisotropy by orbital hybridization with charge transfer in $\left(\mathrm{La}_{0.67} \mathrm{Sr}_{0.33} \mathrm{MnO}_{3}\right)_{n} /$ $\left(\mathrm{SrTiO}_{3}\right)_{n}$ superlattice. NPG Asia Mater, 2018, 10: 931-942

20 Walter J, Bose S, Cabero M, et al. Perpendicular magnetic anisotropy via strain-engineered oxygen vacancy ordering in epitaxial $\mathrm{La}_{1-x} \mathrm{Sr}_{x} \mathrm{CoO}_{3-\delta}$. Phys Rev Mater, 2018, 2: 111404

21 Zhang J, Xue W, Su T, et al. Strain-induced robust magnetic anisotropy and room temperature magnetoelectric coupling effect in epitaxial $\mathrm{SmFeO}_{3}$ film. Sci China Mater, 2020, 63: 2062-2070

22 Chen P, Huang Z, Li C, et al. Binary controls on interfacial magnetism in manganite heterostructures. Adv Funct Mater, 2018, 28: 1801766

23 Guo Z, Lan D, Qu L, et al. Control of ferromagnetism and magnetic anisotropy via tunable electron correlation and spin-orbital coupling in $\mathrm{La}_{0.67} \mathrm{Ca}_{0.33} \mathrm{MnO}_{3} / \mathrm{Ca}(\mathrm{Ir}, \mathrm{Ru}) \mathrm{O}_{3}$ superlattices. Appl Phys Lett, 2018, 113: 231601

24 Chen P, Huang Z, Li M, et al. Enhanced magnetic anisotropy and orbital symmetry breaking in manganite heterostructures. Adv Funct Mater, 2020, 30: 1909536

25 Liao Z, Gauquelin N, Green RJ, et al. Metal-insulator-transition engineering by modulation tilt-control in perovskite nickelates for room temperature optical switching. Proc Natl Acad Sci USA, 2018, 115: 9515-9520

26 Song J, Chen $\mathrm{Y}$, Chen $\mathrm{X}$, et al. Tuning the magnetic anisotropy of $\mathrm{La}_{2 / 3} \mathrm{Sr}_{1 / 3} \mathrm{MnO}_{3}$ by controlling the structure of $\mathrm{SrCoO}_{x}$ in the corresponding bilayers using ionic-liquid gating. Phys Rev Appl, 2019, 12: 054016

27 Zhou G, Wang X, Ji H, et al. The strain induced magnetic and anisotropic variations of $\mathrm{LaCoO}_{3}$ thin films. J Magn Magn Mater, 2020, 515: 167303

28 Ko EK, Mun J, Lee HG, et al. Oxygen vacancy engineering for highly tunable ferromagnetic properties: $\mathrm{A}$ case of $\mathrm{SrRuO}_{3}$ ultrathin film with a $\mathrm{SrTiO}_{3}$ capping layer. Adv Funct Mater, 2020, 30: 2001486

29 Zhang J, Han F, Wang W, et al. Perpendicular magnetic anisotropy in $\mathrm{La}_{1-x} \mathrm{Sr}_{x} \mathrm{CoO}_{2.5+\delta} / \mathrm{La}_{2 / 3} \mathrm{Sr}_{1 / 3} \mathrm{MnO}_{3} / \mathrm{La}_{1-x} \mathrm{Sr}_{x} \mathrm{CoO}_{2.5+\delta}$ trilayers $(x=0.05-$ 0.5). Phys Rev B, 2019, 100: 094432

30 Peng B, Zhou Z, Nan T, et al. Deterministic switching of perpendicular magnetic anisotropy by voltage control of spin reorientation transition in $(\mathrm{Co} / \mathrm{Pt})_{3} / \mathrm{Pb}\left(\mathrm{Mg}_{1 / 3} \mathrm{Nb}_{2 / 3}\right) \mathrm{O}_{3}-\mathrm{PbTiO}_{3}$ multiferroic heterostructures. ACS Nano, 2017, 11: 4337-4345

31 Bruno P. Tight-binding approach to the orbital magnetic moment and magnetocrystalline anisotropy of transition-metal monolayers. Phys Rev B, 1989, 39: 865-868

32 Kresse G, Furthmüller J. Efficient iterative schemes for $a b$ initio totalenergy calculations using a plane-wave basis set. Phys Rev B, 1996, 54: 11169-11186

33 Anisimov VI, Zaanen J, Andersen OK. Band theory and Mott insulators: Hubbard $U$ instead of Stoner I. Phys Rev B, 1991, 44: 943-954

34 Perdew JP, Chevary JA, Vosko SH, et al. Atoms, molecules, solids, and surfaces: Applications of the generalized gradient approximation for exchange and correlation. Phys Rev B, 1992, 46: 6671-6687

35 Son J, LeBeau JM, Allen SJ, et al. Conductivity enhancement of ultrathin $\mathrm{LaNiO}_{3}$ films in superlattices. Appl Phys Lett, 2010, 97: 202109

36 Liao Z, Skoropata E, Freeland JW, et al. Large orbital polarization in nickelate-cuprate heterostructures by dimensional control of oxygen coordination. Nat Commun, 2019, 10: 589

37 Gibert M, Viret M, Torres-Pardo A, et al. Interfacial control of magnetic properties at $\mathrm{LaMnO}_{3} / \mathrm{LaNiO}_{3}$ interfaces. Nano Lett, 2015, 15 : 7355-7361

38 Dieny B, Chshiev M. Perpendicular magnetic anisotropy at transition metal/oxide interfaces and applications. Rev Mod Phys, 2017, 89: 025008

39 Kumar D, David A, Fouchet A, et al. Strong magnetic anisotropy of epitaxial $\mathrm{PrVO}_{3}$ thin films on $\mathrm{SrTiO}_{3}$ substrates with different orientations. ACS Appl Mater Interfaces, 2020, 12: 35606-35613

40 Huang $\mathrm{K}, \mathrm{Wu} \mathrm{L}$, Wang $\mathrm{M}$, et al. Tailoring magnetic order via atomically stacking $3 d / 5 d$ electrons to achieve high-performance spintronic devices. Appl Phys Rev, 2020, 7: 011401

$41 \mathrm{Lu}$ J, Si L, Yao X, et al. Electric field controllable high-spin $\mathrm{SrRuO}_{3}$ driven by a solid ionic junction. Phys Rev B, 2020, 101: 214401

42 Lu J, Si L, Zhang Q, et al. Defect-engineered Dzyaloshinskii-Moriya interaction and electric-field-switchable topological spin texture in $\mathrm{SrRuO}_{3}$. Adv Mater, 2021, 33: 2102525

43 Cui Z, Grutter AJ, Zhou H, et al. Correlation-driven eightfold magnetic anisotropy in a two-dimensional oxide monolayer. Sci Adv, 2020, 6: eaay0114

44 Coughlin AL, Xie D, Yao Y, et al. Near degeneracy of magnetic phases in two-dimensional chromium telluride with enhanced perpendicular magnetic anisotropy. ACS Nano, 2021, 14: 15256-15266

45 Huijben M, Koster G, Liao ZL, et al. Interface-engineered oxygen octahedral coupling in manganite heterostructures. Appl Phys Rev, 2017, 4: 041103

46 Zhou G, Ji H, Bai $\mathrm{Y}$, et al. Intrinsic exchange bias effect in strainengineered single antiferromagnetic $\mathrm{LaMnO}_{3}$ films. Sci China Mater, 2019, 62: 1046-1052

47 Pesquera D, Herranz G, Barla A, et al. Surface symmetry-breaking and strain effects on orbital occupancy in transition metal perovskite epitaxial films. Nat Commun, 2012, 3: 1189

48 Nakao H, Sudayama T, Kubota M, et al. Magnetic and electronic states in $\left(\mathrm{LaMnO}_{3}\right)_{2}\left(\mathrm{SrMnO}_{3}\right)_{2}$ superlattice exhibiting a large negative magnetoresistance. Phys Rev B, 2015, 92: 245104

49 Chen X, Zhang S, Liu B, et al. Theoretical investigation of magnetic anisotropy at the $\mathrm{La}_{0.5} \mathrm{Sr}_{0.5} \mathrm{MnO}_{3} / \mathrm{LaCoO}_{2.5}$ interface. Phys Rev B, 2019, 100: 144413

Acknowledgements This work was financially supported by the National Natural Science Foundation of China $(51901118,51871137,12174237$, and 52171183), the 1331 Engineering of Shanxi Province, the Research Project Supported by Shanxi Scholarship Council of China (2021-093), and the Scientific and Technological Innovation Programs of Higher Education In- 
stitutions in Shanxi (2020L0237). We acknowledge the XAS measurement at Beamline BL08U1A in Shanghai Synchrotron Radiation Facility (SSRF) and the XLD measurement at Beamline BL12-a in National Synchrotron Radiation Laboratory (NSRL). We thank Dr. Z. Quan and Dr. W. Lin for helpful discussions.

Author contributions Zhou $\mathrm{G}$ and Ji $\mathrm{H}$ designed the research and analyzed the data. Ji $\mathrm{H}$ grew the samples and carried out the magnetism and transport measurements. Yan Z performed the first-principles calculations. Cai M, Lu $\mathrm{J}$, and Zhang JX helped to characterize the magnetic domain. Kang $\mathrm{P}$ and Zhang J contributed to the XAS and XLD measurements. Zhou G, Chen J, and $\mathrm{Xu} \mathrm{X}$ discussed the results and commented on the manuscript.

Conflict of interest The authors declare that they have no conflict of interest.

Supplementary information Supporting data are available in the online version of the paper.

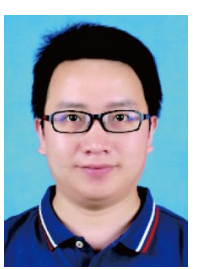

Guowei Zhou obtained his $\mathrm{PhD}$ degree from Shanxi Normal University in 2018. He was a research fellow at the National University of Singapore in 2019. He is currently an associate professor in Prof. Xu's group at Shanxi Normal University. His current research interest focuses on emerging phenomena and exotic physical behaviors in perovskite complex oxides films and heterostructures.

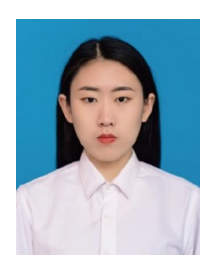

Huihui Ji obtained her Bachelor's degree from the School of Chemistry and Materials Science, Shanxi Normal University in 2016. After that, she continued her education as a $\mathrm{PhD}$ candidate under the supervision of Prof. Xiaohong $\mathrm{Xu}$ at Shanxi Normal University. Her research focuses on the interface phenomena in the strongly correlated oxide heterostructures.

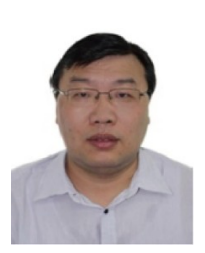

Jingsheng Chen received his $\mathrm{PhD}$ degree from Lanzhou University, China, in 1999. He joined Nanyang Technological University as a postdoctor in 1999-2001 and the Data Storage Institute, A*STAR as a research scientist in 2001-2007. In the year of 2007, he joined the National University of Singapore as an assistant professor and was promoted to associate professor in 2013. His research interest includes high-anisotropy magnetic materials, spintronics, multiferroic materials, and nanostructured magnetic materials.

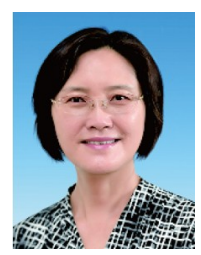

Xiaohong Xu received her $\mathrm{PhD}$ degree in materials science and engineering from Xi'an Jiaotong University, China in 2001. From 2001 to 2006, she worked as a postdoc or research fellow at Huazhong University of Science and Technology (China), the University of Sheffield (UK), and Tohoku University (Japan). Her research interest includes oxide semiconductor spintronics, magnetic recording media, and interface physics of heterostructures.

\section{界面工程对低维锰氧化物超晶格中磁各向异性的调 控}

周国伟 ${ }^{1,2 \dagger}$, 姬慧慧 ${ }^{1 \dagger}$, 严志 ${ }^{1}$, 蔡米铭 $^{4}$, 康鹏华 ${ }^{1}, 弓^{\circ}$ 军 $^{1}$, 鲁京迪 ${ }^{4}$, 张金星 ${ }^{4}$, 陈景升 $^{3 *}$, 许小红 ${ }^{1,2}$

摘要 低维系统磁各向异性的调控无论对于基础研究领域还是对于器 件的工业化应用都有着重要的意义. $\mathrm{La}_{0.7} \mathrm{Sr}_{0.3} \mathrm{MnO}_{3}$ (LSMO) 展现出的高 居里温度以及铁磁半金属特性, 吸引了大量的科研人员对其磁各向异 性进行研究. 但直到目前为止, 关于低维LSMO薄膜中磁各向异性与其 电子结构之间的关系尚不明确. 本文中, 我们利用脉冲激光沉积系统制 备了高质量、原子级平整的 $\mathrm{LSMO} / \mathrm{SrMnO}_{3}(\mathrm{SMO})$ 超晶格. 磁性及电输 运实验结果表明, 随着SMO厚度的增加, 超晶格的磁性易轴由面内转向 面外方向, 并且在一定厚度时, 超晶格的磁各向异性显示出特殊的四重 对称性. X射线线性二色谱及第一性原理计算表明, 超晶格中磁各向异 性的变化与界面处轨道重构有关: 随着SMO厚度的增加, 超晶格中电子 将由择优占据面内轨道转变为择优占据面外轨道. 本实验提出了一种 调控磁各向异性的新方法, 为异质结中磁性质的控制及新现象的产生 提供了新的研究思路. 